\title{
Tunable Persistent Random Walk in Swimming Droplets
}

\author{
Adrien Izzet $\odot,{ }^{1}$ Pepijn G. Moerman $\odot,{ }^{1,2}$ Preston Gross, ${ }^{1}$ Jan Groenewold $\odot,{ }^{2,3}$ \\ Andrew D. Hollingsworth, ${ }^{1}$ Jérôme Bibette, ${ }^{4}$ and Jasna Brujic ${ }^{1, *}$ \\ ${ }^{1}$ Center for Soft Matter Research, Physics Department, New York University, \\ New York, New York 10003, USA \\ ${ }^{2}$ Debye Institute for Nanomaterials, Utrecht University, 3584 Utrecht, The Netherlands \\ ${ }^{3}$ Guangdong Provincial Key Laboratory of Optical Information Materials and Technology \\ and Institute of Electronic Paper Displays, South China Academy of Advanced Optoelectronics, \\ South China Normal University, Guangzhou 510006, People's Republic of China \\ ${ }^{4}$ Laboratoire Colloïdes et Matériaux Divisés, CNRS UMR 8231, Chemistry Biology \& Innovation, \\ ESPCI Paris, PSL Research University, 10 rue Vauquelin, 75005 Paris, France
}

(Received 28 August 2019; revised manuscript received 22 January 2020; accepted 25 March 2020; published 14 May 2020)

\begin{abstract}
We characterize the motility of athermal swimming droplets within the framework of a persistent random walk. Just like active colloids, their trajectories can be modeled with a constant velocity $V$ and a slow angular diffusion, but the random changes in direction are not thermally driven. Instead, $V$ is determined by the interfacial tension gradient along the droplet surface, while reorientation of the surfactant gradient leads to changes in direction with a persistence time $\tau$. We show that the origin of locomotion is the difference in the critical micellar concentration in the front and the back of the droplet, $\triangle \mathrm{CMC}$. Tuning this parameter by salt controls $V$ from 3 to 15 diameters $d / s$. Surfactant concentration has little effect on speed, but leads to a dramatic decrease in $\tau$ over 4 orders of magnitude. The corresponding range of the persistence length $\ell=V \tau$ extends beyond the realm of synthetic or living swimmers, in which $V$ is limited by fuel consumption and $\tau$ is set by thermal fluctuations or biological activity, respectively. Our tunable swimmers are ideal candidates for the study of the departure from equilibrium to high levels of activity. We show that their collective behavior exhibits the formation of active clusters of a well-defined size.
\end{abstract}

DOI: 10.1103/PhysRevX.10.021035

Most active particles, such as Janus colloids [1], are anisotropic and their orientation determines the swimming direction. They consume fuel preferentially on one side of the particle, which results in chemical gradients that drive and direct self-propulsion. On short timescales, these swimmers move in a straight line, but they are small enough that thermal diffusion randomizes the swimming direction on long timescales. The transition between these two regimes is characterized by the persistence time, and they are modeled as active Brownian particles [2,3]. It is difficult to control how far the active particles are from equilibrium because the velocity and persistence time are coupled quantities.

*jb2929@nyu.edu; https://wp.nyu.edu/brujiclab/

Published by the American Physical Society under the terms of the Creative Commons Attribution 4.0 International license. Further distribution of this work must maintain attribution to the author(s) and the published article's title, journal citation, and DOI.
Subject Areas: Chemical Physics, Soft Matter,

Statistical Physics
Unlike Janus particles, active droplets are chemically isotropic, yet they spontaneously break symmetry as they dissolve to create swollen micelles [4]. At a sufficiently large Péclet number, the isotropic state becomes unstable to small perturbations, resulting in self-sustained motion in a given direction [5]. Autochemotaxis of the droplet is explained by an interfacial gradient along the surface, which induces a Marangoni stress and drives the flow of surfactants. It is thought that droplet motion itself is sufficient to cause the asymmetry needed to sustain a surfactant gradient, as the droplet encounters empty micelles in the front and leaves filled ones at the back $[5,6]$. This Marangoni effect is at play in all droplet swimmers: direct emulsions made of oil [7] or nematic liquid crystals [8-11], as well as inverse emulsions [12,13]. Depending on their size and fuel concentration, previous studies have empirically characterized trajectories of ordinary emulsions as ballistic (straight) or diffusive (random) $[11,13]$, and those of liquid crystal droplets as helical [9] and even oscillatory $[11,14]$. Because swimming droplets are typically athermal, ranging in size from 10 to $300 \mu \mathrm{m}$, their reorientation cannot be a thermally driven process and 
their motility is proposed to be different from that of active colloids $[15,16]$. The direction of droplet motion is fixed by the orientation of the surfactant gradient, which varies in time on timescales that are significantly faster than their thermal diffusion timescale. Despite all these advances, a quantitative analysis of the motility profiles as a function of experimental control parameters, as well as their theoretical interpretation, is still lacking in the literature.

In this paper, we first present an alternative origin for the driving force of Marangoni flows in direct emulsions to that proposed in Ref. [5], which relies on the difference between the critical micellar concentration (CMC) of the surfactant in pure water and in the presence of oil, $\triangle \mathrm{CMC}$. Second, we demonstrate that droplet velocity $V$ and persistence time $\tau$ can be independently tuned by the salt and surfactant concentration, respectively. Third, we show that the singleparticle dynamics is well fit with a persistent random walk, in which the trend in velocity is quantitatively explained by theory, while the measured rotational diffusion timescales significantly deviate from purely thermal diffusion. Since the microscopic mechanism that underlies turning is active rather than thermal, we are able to tune $\tau$ over 4 orders of magnitude. Swimming droplets therefore explore a wider range of parameters than active colloids or bacteria $[17,18]$. This is important because the persistence length $\ell=V \tau$ is the natural control parameter of departure from equilibrium [19-21]. For instance, we finally show that placing many droplets together in close contact leads to the stabilization of active clusters, as a consequence of the balance between depletion attraction and repulsive active interactions. This system opens the possibility to study motility induced phase separation (MIPS), which should occur for particles with a long persistence length at high levels of activity [22-24]. While the persistent random walk model holds for small droplets $<50 \mu \mathrm{m}$, we note that more exotic effects, including self-avoidance [6] and oscillations [11], are observed for larger emulsions, and are beyond the scope of this study.

Droplets of diethyl phthalate (DEP) are produced in a custom-built glass microfluidic setup with a coaxial coflow geometry [25-27]. The continuous phase is initially a $10 \mathrm{mM}$ sodium dodecyl sulphate (SDS) solution saturated with DEP to prevent autophoretic motion and stabilize the droplets. To initiate swimming, $0.5 \mu \mathrm{L}$ of the emulsion is injected using a micropipette into a circular sample cell ( $1 \mathrm{~cm}$ wide and $1 \mathrm{~mm}$ deep), filled with SDS solution at the desired concentration. Droplet trajectories are observed using a Nikon Eclipse Ti microscope equipped with a $4 \times$ Plan Apochromat $\lambda$ Nikon objective $(\mathrm{NA}=0.20)$. The movies are recorded using a Zyla sCMOS Andor camera operating at 40 frames/s. To compare droplets of the same size, we crop the trajectories in a time window corresponding to a droplet diameter of $30 \pm 5 \mu \mathrm{m}$.

Our system therefore consists of droplets of DEP suspended in an aqueous solution of SDS. This oil is slightly
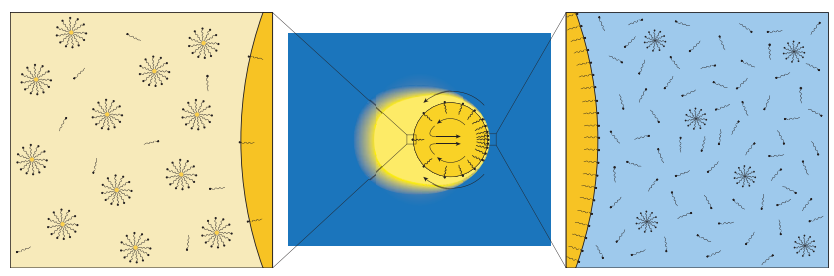

FIG. 1. Schematic of the mechanism at the origin of selfsustained droplet motion. Spontaneous symmetry breaking arises due to a higher concentration of monomers in equilibrium with the aqueous solution (on the right) compared to those facing DEP-saturated water (on the left). This effect arises because of the difference in the $\mathrm{CMC}$ in the two environments, and it causes a Marangoni flow, shown by the arrows, which is enhanced by droplet motion.

soluble in water $(1 \mathrm{mM})$, which lowers the CMC of SDS from $8 \mathrm{mM}$ in pure water to $5 \mathrm{mM}$ in the vicinity of the droplets. This decrease corresponds to a decrease in $\Delta G_{\text {mic }}=$ $2.3 k_{B} T \log (\Delta \mathrm{CMC})=1.1 k_{B} T$ for the micellization energy of one monomer of SDS [28,29]. The resulting DEP swollen micelles are thermodynamically more stable than the empty ones. Therefore, an infinitesimal perturbation in the velocity of the droplet leads to an anisotropy in surfactant concentration, on the order of $\triangle \mathrm{CMC}$, on the droplet interface: the part facing DEP-saturated water sheds more surface active monomers into solution than the part facing the empty micelles in the bulk, as shown in Fig. 1. This asymmetry induces a Marangoni stress that forces the surface flow of surfactants. At high enough dissolution rates compared to molecular diffusion, the interfacial gradient undergoes a swimming instability and the droplets self-propel [5]. More quantitatively, Fig. 2 shows droplet trajectories from which we extract instantaneous droplet velocities $\vec{V}$ over
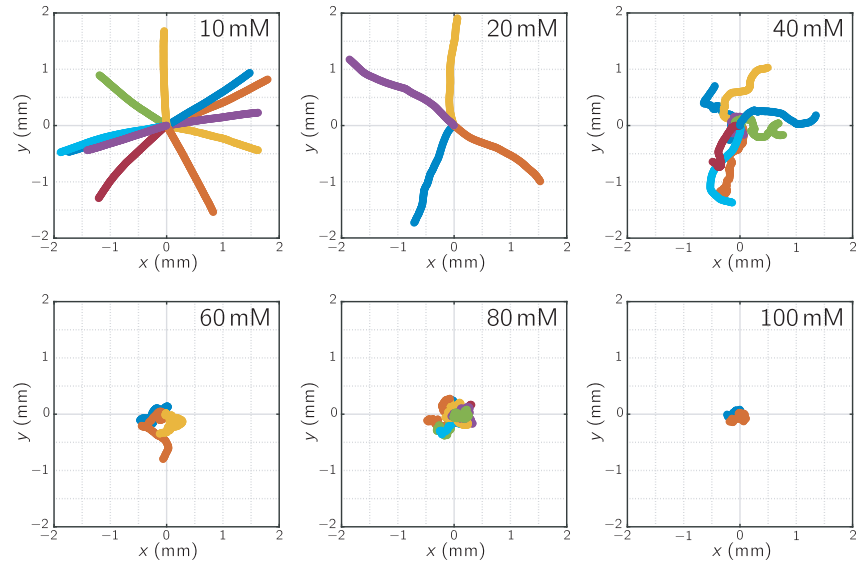

FIG. 2. Individual trajectories of $30 \mu \mathrm{m}$ diameter droplets, lasting $4 \mathrm{~s}$, swimming in aqueous solutions with increasing bulk SDS concentrations. The motion transitions from ballistic to diffusive, with more frequent turns leading to a slower exploration of space, which is still orders of magnitude higher than that of thermal rotational diffusion. 
time, as shown in Fig. S1 in the Supplemental Material (SM) [30]. Note that this speed decreases slowly over time at a rate of $\approx 10 \mu \mathrm{m} / \mathrm{s}^{2}$, but this effect is negligible over the course of the trajectories. We measure fast speeds up to 15 diameters/s, an order of magnitude faster than any known swimming droplets.

Droplet velocity is given by $\vec{V}=M \vec{\nabla}_{\|} c$, where $M$ is the phoretic mobility and $\nabla_{\|} c$ is the average concentration gradient of the SDS monomer along the surface of the droplet. It has been shown that Marangoni effects are dominant compared to diffusiophoresis in swimming droplets [31]. The velocity is thus given by

$$
\vec{V}=\frac{r}{2 \eta_{0}+3 \eta_{i}}\left(-\frac{\partial \gamma}{\partial c}\right) \vec{\nabla}_{\|}
$$

where $r=15 \mu \mathrm{m}$ is the radius of the droplet, $\eta_{i}=13 \mathrm{mPas}$ is the viscosity of the DEP oil, $\eta_{o}=0.89 \mathrm{mPas}$ is the viscosity of the solute, $\partial \gamma / \partial c$ is the rate of change of surface tension with SDS concentration at the interface, and $\nabla_{\|} c=\Delta \mathrm{CMC} / 2 r$ is controlled by $\Delta \mathrm{CMC}$.

Using the pendant drop method, we measure the surface tension as a function of surfactant concentration, in the presence and absence of DEP in the aqueous phase, and find the difference in the $\mathrm{CMC}$ to be $\triangle \mathrm{CMC}=3 \mathrm{mM}$ in Fig. 3. Note that the DEP oil drop in pure water is

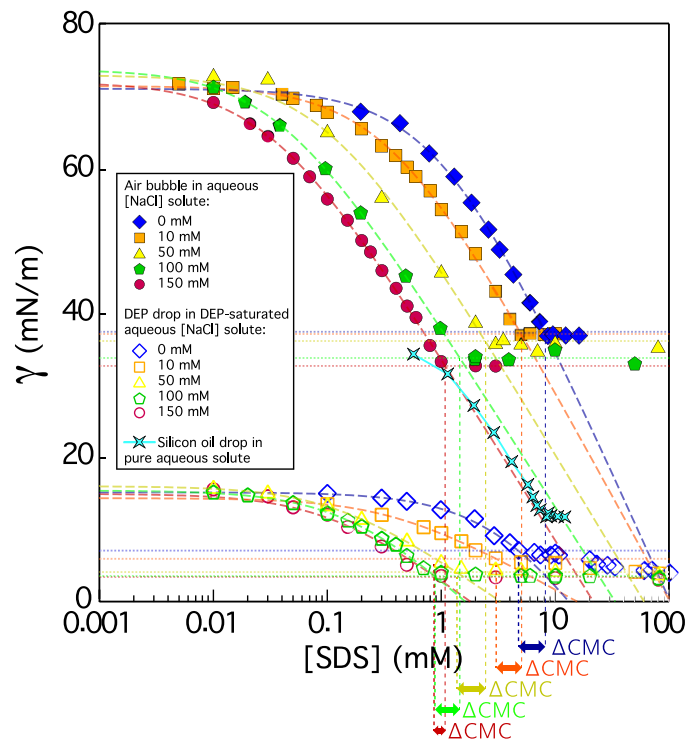

FIG. 3. Surface tension versus SDS concentration at the interface of an air bubble in aqueous $[\mathrm{NaCl}]$ solution (filled symbols), a DEP drop in DEP-saturated $[\mathrm{NaCl}]$ aqueous solution (open symbols), and a silicone oil drop (350 centiStokes) in pure aqueous solution (crosses). As a function of $[\mathrm{NaCl}]$, fits to the von Szyszkowski equation (dashed lines) reveal that the surface tension gradient is independent of salt concentration for a given interface. The CMC decreases with salt for both the aqueous and the DEP-saturated interface, but the difference $\triangle \mathrm{CMC}$ is progressively smaller. unstable because the DEP continuously dissolves and alters the surface tension measurement. We therefore replace the DEP drop with an air bubble in these measurements. The fact that air and silicone oil in water have similar surface tension gradients and CMC values, also shown, supports the assumption that the DEP-water interface in our experiments behaves similarly. For the experimentally determined speed $V \sim 450 \mu \mathrm{m} / \mathrm{s}$, shown in Fig. 4, we then calculate $\partial \gamma / \partial c=0.01 \mathrm{~N}(\mathrm{~m} \mathrm{M})^{-1}$. Comparing this value to the tension gradient in the range of $\triangle \mathrm{CMC}$ at the interface with pure water [30], we find that it is 2 orders of magnitude smaller than the expected value. This discrepancy is consistent with the numerical prediction of Ref. [5] that one can only achieve $1 \%-10 \%$ of the theoretical swimming speed in the Janus limit, depending on the Péclet number that relates the advective and diffusive surfactant transport. Alternatively, if the process is modeled as an adsorption at the interface, such that $-\nabla \gamma=k_{B} T \lambda \nabla c$ $[13,31]$, the measured speed predicts an interaction length $\lambda=5 \mathrm{~nm}$, which is in agreement with the diameter of a SDS micelle of $3.7 \mathrm{~nm}[32,33]$.

To test the hypothesis that the driving force for propulsion is the $\triangle \mathrm{CMC}$ at the interface, we add sodium chloride $(\mathrm{NaCl})$ to decrease $\triangle \mathrm{CMC}[35]$ and measure the resulting velocity at a fixed surfactant concentration of $10 \mathrm{mM}$. First, we estimate $\triangle \mathrm{CMC}$ by fitting the surface tension as a function of SDS and salt concentration using the equation proposed in Ref. [36] to reveal a gradual decrease in the CMC in both pure and DEP-saturated water in Fig. 3. The

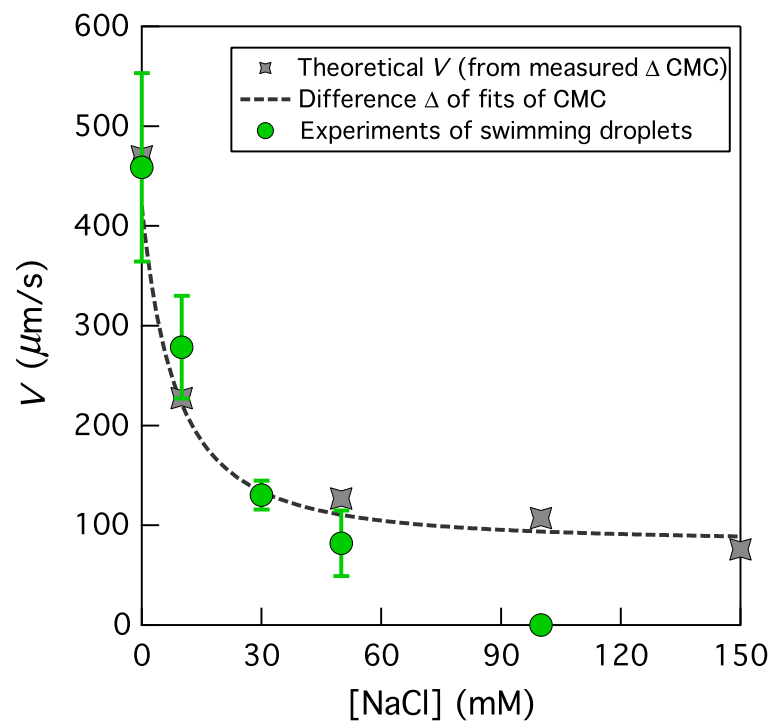

FIG. 4. Instantaneous speed $V$ at a constant $[\mathrm{SDS}]=10 \mathrm{mM}$ sharply decreases with $[\mathrm{NaCl}]$. This trend is theoretically captured by Eq. (1) with $\triangle \mathrm{CMC}$ values independently measured by pendant drop experiments. Above $[\mathrm{NaCl}]=70 \mathrm{mM}$, corresponding to $\triangle \mathrm{CMC}=0.5 \mathrm{mM}$, droplets cease to swim. Dashed line is computed from the difference between empirical fits of $\mathrm{CMC}$ versus [SDS] [34]). 
difference in the $\mathrm{CMC}$ decreases because the $\mathrm{CMC}$ decays faster in pure water.

Assuming all other terms in Eq. (1) remain the same, the change in $\triangle \mathrm{CMC}$ accurately predicts the sharp decrease in the average instantaneous speed by adding salt, as shown in Fig. 4. This assumption is corroborated by the fact that the gradients $\partial \gamma / \partial c$ below the CMC (see Fig. 3) are largely independent of salt. If we consider the surface adsorption picture, results are also consistent since the micelle size, which sets the characteristic length scale $\lambda$, hardly changes as a function of salt [33]. An important difference between experiment and theory is that droplets do not swim below $\Delta \mathrm{CMC} \approx 0.5 \mathrm{mM}$, while the theoretical prediction approaches a constant speed. This result is consistent with the theoretical prediction that self-sustained autophoresis takes place only above a threshold Péclet number of Pe $>4$, when the advection is strong enough [5]. This corresponds to a minimum $\triangle \mathrm{CMC}$, below which swimming is not sustained. In Fig. S2 in SM [30], we extend the study to $\mathrm{LiCl}$, which affects the $\triangle \mathrm{CMC}$ of SDS more abruptly than $\mathrm{NaCl}$, in agreement with a sharper decay in the velocity with $\mathrm{LiCl}$ concentration [37,38]. Direct control of the speed via $\triangle \mathrm{CMC}$ not only presents a useful toolbox, but also lends support to the idea that micellar equilibration at the interface is at the origin of Marangoni flows. The model proposed here is general and may apply to experimental data from other groups $[6,13]$.

Next, we investigate the effect of adding surfactants on the motility of individual droplets. Representative trajectories over $4 \mathrm{~s}$ are shown for various SDS concentrations in Fig. 2. Slightly above the CMC, droplets swim with a constant speed in a given direction. As the concentration increases, droplets change their direction more frequently, and therefore explore less space. We measure the persistence time from the characteristic decorrelation time of the velocity-velocity correlation function (VCF) averaged over the whole trajectory [39]:

$$
C(\Delta t)=\left\langle\frac{\vec{v}(t) \cdot \vec{v}(t+\Delta t)}{|\vec{v}(t)||\vec{v}(t+\Delta t)|}\right\rangle_{t} .
$$

In Fig. 5, also shown on a semilog plot in Fig. S3 in SM [30], we demonstrate that the data are fit well with an exponential decay, $C(\Delta t)=\exp (-\Delta t / \tau)$, to obtain the characteristic times over which the swimming direction randomizes. The trend of $\tau$ with SDS concentration is shown in Fig. 7(b).

To quantify the droplet motility, we next compute the mean square displacement (MSD) $\Delta L^{2}$ of the trajectories, shown on a log-log plot in Fig. 6. We find that swimming droplets are ballistic at short times, where the slope is two, and they become diffusive at long times, where the slope is one. The crossover time $\tau$ between the two regimes decreases with increasing SDS concentration. These results are fit by the persistent random walk model [40],

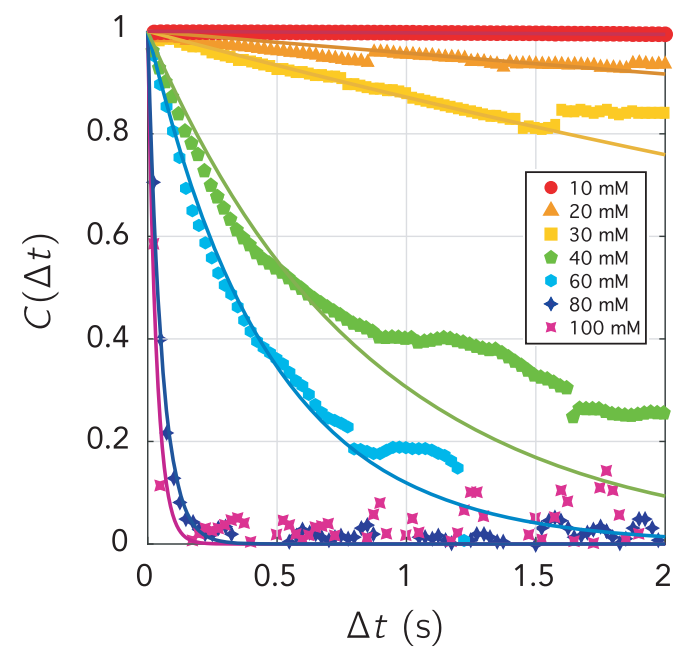

FIG. 5. Normalized autocorrelation of the velocity for each concentration. Each dataset is computed from averaging the $C(\Delta t)$ of experimental trajectories at a given concentration. Solid lines are fit with an exponential decay following Eq. (2).

$$
\Delta L^{2}=2 \tau^{2} V^{2}\left[\frac{\Delta t}{\tau}+\exp \left(-\frac{\Delta t}{\tau}\right)-1\right]
$$

where the fitting parameters $V$ and $\tau$ are shown in Figs. 7(a) and 7 (b), respectively. We find that $V$ is largely independent of surfactant concentration, since $\triangle \mathrm{CMC}$ is independent of surfactant concentration. This result is in good agreement with direct measurements of the average instantaneous speed along the trajectories, as well as previous measurements on small droplets [11]. These results imply that droplet speed is also independent of the dissolution rate, which increases with SDS concentration, as shown in

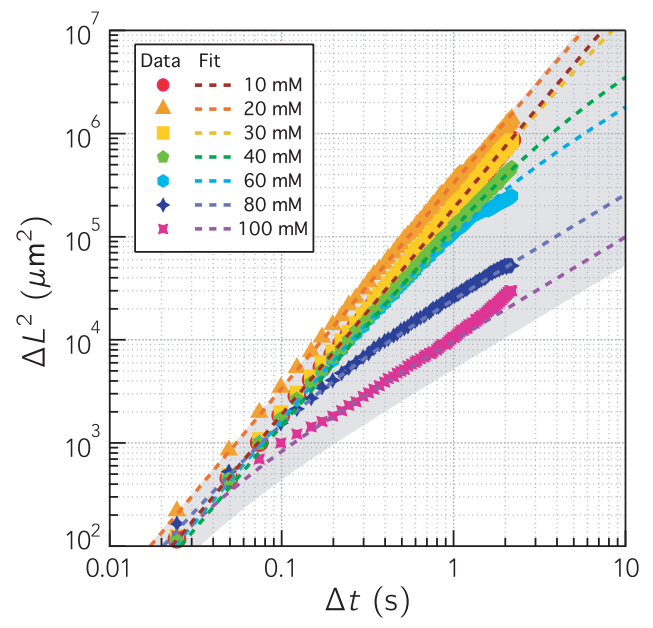

FIG. 6. Mean square displacement curves, averaged over tens of trajectories, show a decrease in the crossover time $\tau$ as a function of SDS concentration. The model of rotational diffusion in Eq. (3) gives an excellent fit to the data and the gray area corresponds to the $95 \%$ confidence interval. 



FIG. 7. Effect of surfactant concentration on motility. (a) Instantaneous speed $V$, estimated directly from trajectories (circles) or the MSD fits of Eq. (3) (squares), is largely independent of [SDS]. (b) Persistence time $\tau$, from the fits and the values extracted from the velocity correlation function $C(\Delta t)$, sharply decays with [SDS] with a heuristic power-law fit $\tau=6 \times 10^{6}[\mathrm{SDS}]^{-4}$. (c) The rate of droplet dissolution increases nonlinearly as a function of SDS concentration over the experimental range.

Fig. 7(c). This result suggests that the equilibration timescale of the monomers at the interface with the bulk is faster than micellar solubilization. Droplet locomotion is governed by $\triangle \mathrm{CMC}$ and not the bulk micellar concentration, in support of the proposed mechanism for propulsion in Fig. 1.

Fits of the MSD also show a decrease in $\tau$ with surfactant concentration that is dramatic: over one decade of SDS concentration, the fitting parameter $\tau$ in Eq. (3) decreases over 4 orders of magnitude, giving rise to a power-law exponent of -4 in the fit in Fig. 7(b). This estimate of $\tau$ alone is unreliable because the trajectories are much shorter than the persistence time, but the excellent agreement with $\tau$ obtained from the VCF fits in Fig. 5 confirm the trend that droplets turn more frequently as more surfactant is added.

This result differs from other active colloids, in which the persistence time is tuned by the particle size [41], or by biological processes in the case of living organisms [18]. This limits the range of reported $\tau$ to $0.1-1 \mathrm{~s}$ for colloids with thermally activated particle sizes. If the angular diffusion of $30 \mu \mathrm{m}$ droplets were thermal, their persistence time would be $\approx 6 \mathrm{~h}$, corresponding to a diffusion constant of $D_{r}=1 / \tau=0.00005 \mathrm{~s}^{-1}$. Here, we find effective rotational diffusion constants up to $D_{r}=100 \mathrm{~s}^{-1}$.

The statistics of this active turning process are captured by the distributions of the instantaneous speed and reorientation angle as a function of SDS concentration in Fig. 8. For a given $\delta t=25 \mathrm{~ms}$, the displayed position at time $t+\delta t$ along the trajectory informs on both the speed and change in direction with respect to the previous time step. Since the position at time $t$ lies at the origin, positions on the positive $x$ axis indicate straight motion, while particles that turn result in an off-axis position. As the SDS concentration increases, the characteristic time $\tau$ decreases to approach $\delta t$. When $\tau \gg \delta t$, we observe ballistic trajectories focused on a single point in Fig. 8(a), where the noise is on the order of a pixel size. Intermediate concentrations give rise to noise in both speed and reorientation angle, but the droplets rarely turn back on themselves [Fig. 8(b)]. Eventually, at $\tau \approx \delta t$ droplets sample the space with equal

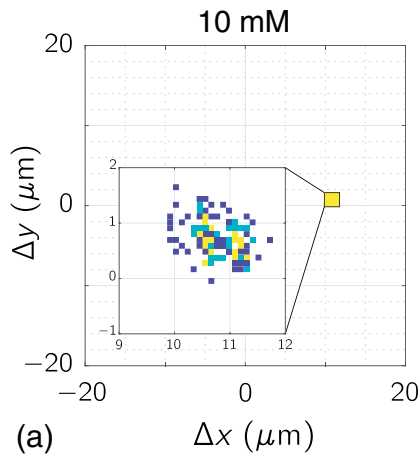

(a)
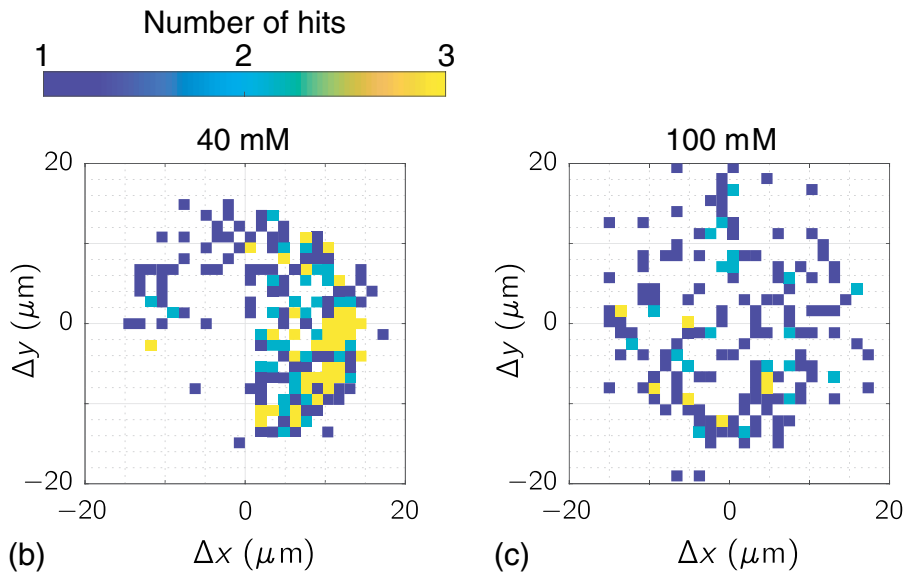

FIG. 8. Displacements of the droplet between consecutive time steps (i.e., $\Delta t=25 \mathrm{~ms}$ ), for three surfactant concentrations. Colors represent the density at which this location in the displacement field is hit along the trajectory. 


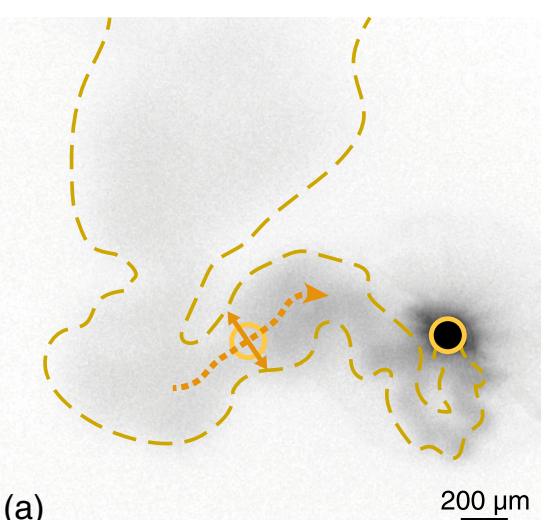

(a)
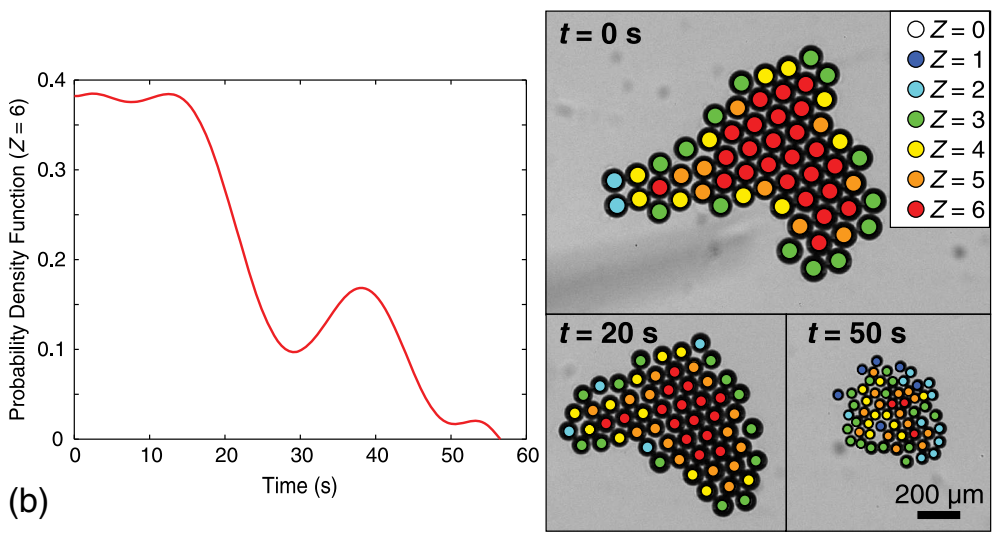

FIG. 9. Memory effects in droplets. (a) Droplets leave a fluorescently labeled trail (dashed lines), which diffusively spreads with a diffusion constant consistent with that of DEP-filled micelles, confirming the proposed mechanism in Fig. 1. (b) Active droplets transition from a crystal to a more dilute, disordered cluster over time. The graph shows the concomitant drop in the coordination number $Z=6$, shown in red in the snapshots from the video.

probability, similar to Brownian diffusion, as indicated by the color map in Fig. 8(c) and in Fig. S4 in SM [30] as a function of SDS concentration.

We attribute this fast stochastic dynamics to active processes at the droplet interface, as described in the recent numerical investigation of the fully coupled hydrodynamic and advection-diffusion problems by Morozov and Michelin [15]. The mobility that dominates the instantaneous velocity of the droplets is driven by the Marangoni effect, quantified in Eq. (1), which induces a symmetric flow within and around the droplet. In addition, droplets undergo a diffusiophoretic mobility mechanism, in which self-generated gradients of surfactants break the symmetry of the surface flow of surfactants. This reorientation of surfactant gradients at the interface causes the droplets to turn in random directions. The diffusiophoretic contribution is enhanced with increasing SDS concentration because the rate of droplet dissolution into micelles increases, as shown in Fig. 7(c), causing sharper gradients surrounding the droplet. This mechanism leads to the dramatic decrease in $\tau$ as a function of SDS concentration. This view is corroborated by Chamolly and Lauga [16], which shows that the ratio between reactive and diffusive fluxes, known as the Damkohler number, governs the transition from ballistic to diffusive trajectories as the reaction rate increases. Further studies on the spatial heterogeneities in the advective flows are needed to quantitatively describe the rapid power-law decay in the persistence time in Fig. 7(b).

These surfactant flows are difficult to visualize at the droplet interface, but it is possible to fluorescently dye the DEP oil, such that the concentration gradient upon micellization becomes visible. In Fig. 9(a), we show the Nile Red trail over the course of the one-minute trajectory. Measuring the width of the trail over time, we find that its diffusion constant is $D=72 \mu \mathrm{m}^{2} / \mathrm{s}$, in excellent agreement with the diffusion constant $D=65 \mu \mathrm{m}^{2} / \mathrm{s}$ of swollen micelles with a diameter of $3.7 \mathrm{~nm}$. This result lends further support to the micellization mechanism as the driving force for droplet propulsion. The droplet is repelled by its own trail, or those of other droplets [7], giving rise to interesting collective behavior.

Starting with a dense configuration of active droplets, we first observe that swimming droplets pack into a crystalline array, rigidly held together by depletion attraction. However, as they dissolve into DEP-filled micelles and induce active repulsive interactions, they disrupt the crystal to form dynamically rearranging clusters of a welldefined size. This phenomenon is shown Fig. 9(b), demonstrating the abrupt decay in the fraction of droplets with coordination number $Z=6$, typical for the triangular lattice structure. This is an example of an order to disorder transition resulting from a competition between thermodynamic and active forces. This is an alternative mechanism for creating and stabilizing "critters" [42], and offers the possibility of cooperative transport.

In this paper, we have presented evidence that the difference in the $\mathrm{CMC}$ at the front and back of the droplet is at the origin of droplet motion, which is why the average instantaneous velocity is independent of surfactant concentration above the $\mathrm{CMC}$, but sensitive to changes in salt. Conversely, the frequency with which droplets change direction drastically increases with surfactant concentration, allowing us to independently tune $V$ and $\tau$ in the experiments. These swimmers broaden the range of active particle sizes to the athermal realm. Such a system promises to be useful in tuning the departure from equilibrium $[21,43]$ and understanding the resulting dynamics $[2,44,45]$. The selfassembly of droplets into equilibrium colloidomers [46] can now be extended to the assembly of nonequilibrium architectures, akin to living systems [47].

The authors gratefully acknowledge insights by Eric Vanden-Eijnden, Katherine Newhall, Alexander Grosberg, Sascha Hilgenfeldt, Willem Kegel, and Alfons Van 
Blaaderen, as well as useful conversations with Micha Kornreich and Rodrigo Guerra. This work was supported by the Materials Research Science and Engineering Center (MRSEC) program of the National Science Foundation under Grants No. NSF DMR-1420073 and in part by Grant No. NSF DMR-1710163.

[1] J. R. Howse, R. A. L. Jones, A. J. Ryan, T. Gough, R. Vafabakhsh, and R. Golestanian, Self-Motile Colloidal Particles: From Directed Propulsion to Random Walk, Phys. Rev. Lett. 99, 048102 (2007).

[2] A. P. Solon, M. E. Cates, and J. Tailleur, Active Brownian Particles and Run-and-Tumble Particles: A Comparative Study, Eur. Phys. J. Special Topics 224, 1231 (2015).

[3] M. E. Cates and J. Tailleur, When Are Active Brownian Particles and Run-and-Tumble Particles Equivalent? Consequences for Motility-Induced Phase Separation, Europhys. Lett. 101, 20010 (2013).

[4] C. A. Weber, D. Zwicker, F. Jülicher, and C. F. Lee, Physics of Active Emulsions, Rep. Prog. Phys. 82, 064601 (2019).

[5] S. Michelin, E. Lauga, and D. Bartolo, Spontaneous Autophoretic Motion of Isotropic Particles, Phys. Fluids 25, 061701 (2013).

[6] C. Jin, C. Krüger, and C.C. Maass, Chemotaxis and Autochemotaxis of Self-Propelling Droplet Swimmers, Proc. Natl. Acad. Sci. U.S.A. 114, 5089 (2017).

[7] P. G. Moerman, H. W. Moyses, E. B. van der Wee, D. G. Grier, A. van Blaaderen, W. K. Kegel, J. Groenewold, and J. Brujic, Solute-Mediated Interactions between Active Droplets, Phys. Rev. E 96, 032607 (2017).

[8] K. Peddireddy, P. Kumar, S. Thutupalli, S. Herminghaus, and C. Bahr, Solubilization of Thermotropic Liquid Crystal Compounds in Aqueous Surfactant Solutions, Langmuir 28, 12426 (2012).

[9] C. Krüger, G. Klös, C. Bahr, and C. C. Maass, Curling Liquid Crystal Microswimmers: A Cascade of Spontaneous Symmetry Breaking, Phys. Rev. Lett. 117, 048003 (2016).

[10] N. Ueno, T. Banno, A. Asami, Y. Kazayama, Y. Morimoto, T. Osaki, S. Takeuchi, H. Kitahata, and T. Toyota, SelfPropelled Motion of Monodisperse Underwater Oil Droplets Formed by a Microfluidic Device, Langmuir 33, 5393 (2017).

[11] M. Suga, S. Suda, M. Ichikawa, and Y. Kimura, SelfPropelled Motion Switching in Nematic Liquid Crystal Droplets in Aqueous Surfactant Solutions, Phys. Rev. E 97, 062703 (2018).

[12] S. Thutupalli, R. Seemann, and S. Herminghaus, Swarming Behavior of Simple Model Squirmers, New J. Phys. 13, 073021 (2011).

[13] Z. Izri, M. N. Van Der Linden, S. Michelin, and O. Dauchot, Self-Propulsion of Pure Water Droplets by Spontaneous Marangoni-Stress-Driven Motion, Phys. Rev. Lett. 113, 248302 (2014).

[14] S. Herminghaus, C. C. Maass, C. Krüger, S. Thutupalli, L. Goehring, and C. Bahr, Interfacial Mechanisms in Active Emulsions, Soft Matter 10, 7008 (2014).
[15] M. Morozov and S. Michelin, Nonlinear Dynamics of a Chemically-Active Drop: From Steady to Chaotic SelfPropulsion, J. Chem. Phys. 150, 044110 (2019).

[16] A. Chamolly and E. Lauga, Stochastic Dynamics of Dissolving Active Particles, Eur. Phys. J. E 42, 88 (2019).

[17] J. Saragosti, P. Silberzan, and A. Buguin, Modeling E. coli Tumbles by Rotational Diffusion. Implications for Chemotaxis, PLoS One 7, e35412 (2012).

[18] A. E. Patteson, A. Gopinath, M. Goulian, and P. E. Arratia, Running and Tumbling with E. coli in Polymeric Solutions, Sci. Rep. 5, 15761 (2015).

[19] J. Tailleur and M. E. Cates, Sedimentation, Trapping, and Rectification of Dilute Bacteria, Europhys. Lett. 86, 60002 (2009).

[20] C. Bechinger, R. Di Leonardo, H. Löwen, C. Reichhardt, G. Volpe, and G. Volpe, Active Particles in Complex and Crowded Environments, Rev. Mod. Phys. 88, 045006 (2016).

[21] É. Fodor, C. Nardini, M. E. Cates, J. Tailleur, P. Visco, and F. Van Wijland, How Far from Equilibrium Is Active Matter?, Phys. Rev. Lett. 117, 038103 (2016).

[22] G. S. Redner, M. F. Hagan, and A. Baskaran, Structure and Dynamics of a Phase-Separating Active Colloidal Fluid, Phys. Rev. Lett. 110, 055701 (2013).

[23] M. E. Cates and J. Tailleur, Motility-Induced Phase Separation, Annu. Rev. Condens. Matter Phys. 6, 219 (2015).

[24] J. U. Klamser, S. C. Kapfer, and W. Krauth, Thermodynamic Phases in Two-Dimensional Active Matter, Nat. Commun. 9, 5045 (2018).

[25] C. Cramer, P. Fischer, and E. J. Windhab, Drop Formation in a Co-Flowing Ambient Fluid, Chem. Eng. Sci. 59, 3045 (2004).

[26] C. H. Choi, D. A. Weitz, and C. S. Lee, One Step Formation of Controllable Complex Emulsions: From Functional Particles to Simultaneous Encapsulation of Hydrophilic and Hydrophobic Agents into Desired Position, Adv. Mater. 25, 2536 (2013).

[27] M. F. Haase and J. Brujic, Tailoring of High-Order Multiple Emulsions by the Liquid-Liquid Phase Separation of Ternary Mixtures, Angew. Chem. Int. Ed. 53, 11793 (2014).

[28] J. N. Israelachvili, Intermolecular and Surface Forces, 3rd ed. (Academic Press, New York, 2011).

[29] M. J. Rosen and J. T. Kunjappu, Surfactants and Interfacial Phenomena: Fourth Edition (John Wiley \& Sons Inc., New York, 2012).

[30] See Supplemental Material at http://link.aps.org/ supplemental/10.1103/PhysRevX.10.021035 for a more detailed analysis of swimming trajectories.

[31] J. Anderson, Colloid Transport by Interfacial Forces, Annu. Rev. Fluid Mech. 21, 61 (1989).

[32] S. Hayashi and S. Ikeda, Micelle Size and Shape of Sodium Dodecyl Sulfate in Concentrated Sodium Chloride Solutions, J. Phys. Chem. 84, 744 (1980).

[33] G. Duplâtre, M.F. F. Marques, and M. da Graça Miguel, Size of Sodium Dodecyl Sulfate Micelles in Aqueous Solutions as Studied by Positron Annihilation Lifetime Spectroscopy, J. Phys. Chem. 100, 16608 (1996).

[34] S. A. Morton, D. J. Keffer, A. N. Davis, and R. M. Counce, Effect of Low Concentration Salt on Organic Contact Angle 
in Ionic Surfactant Solutions: Insight from Theory and Experiment, Sep. Sci. Technol. 43, 310 (2008).

[35] B. Principles, Emulsion Science, Springer Tracts in Modern Physics, Vol. 181 (Springer, Berlin, 2002).

[36] B. von Szyszkowski, Experimental Studies of the Capillary Properties of Aqueous Solutions of Fatty Acids, Z. Phys. Chem. 64, 385 (1908).

[37] S. S. Berr and R. R. Jones, Effect of Added Sodium and Lithium Chlorides on Intermicellar Interactions and Micellar Size of Aqueous Dodecyl Sulfate Aggregates as Determined by Small-Angle Neutron Scattering, Langmuir 4, 1247 (1988).

[38] C. Maltesh and P. Somasundaran, Effect of Binding of Cations to Polyethylene Glycol on Its Interactions with Sodium Dodecyl Sulfate, Langmuir 8, 1926 (1992).

[39] S. Burov, S. M. A. Tabei, T. Huynh, M. P. Murrell, L. H. Philipson, S. A. Rice, M. L. Gardel, N. F. Scherer, and A. R. Dinner, Distribution of Directional Change as a Signature of Complex Dynamics, Proc. Natl. Acad. Sci. U.S.A. 110, 19689 (2013).

[40] Y. Fily and M. C. Marchetti, Athermal Phase Separation of Self-Propelled Particles with No Alignment, Phys. Rev. Lett. 108, 235702 (2012).
[41] J. Palacci, S. Sacanna, A. P. Steinberg, D. J. Pine, and P. M. Chaikin, Living Crystals of Light-Activated Colloidal Surfers, Science 339, 936 (2012).

[42] M. Driscoll, B. Delmotte, M. Youssef, S. Sacanna, A. Donev, and P. Chaikin, Unstable Fronts and Motile Structures Formed by Microrollers, Nat. Phys. 13, 375 (2017).

[43] G. Gonnella, D. Marenduzzo, A. Suma, and A. Tiribocchi, Motility-Induced Phase Separation and Coarsening in Active Matter, C.R. Phys. 16, 316 (2015).

[44] H. Stark, Artificial Chemotaxis of Self-Phoretic Active Colloids: Collective Behavior, Acc. Chem. Res. 51, 2681 (2018).

[45] S. Thutupalli, D. Geyer, R. Singh, R. Adhikari, and H. A. Stone, Flow-Induced Phase Separation of Active Particles Is Controlled by Boundary Conditions, Proc. Natl. Acad. Sci. U.S.A. 115, 5403 (2018).

[46] A. McMullen, M. Holmes-Cerfon, F. Sciortino, A. Y. Grosberg, and J. Brujic, Freely Jointed Polymers Made of Droplets, Phys. Rev. Lett. 121, 138002 (2018).

[47] Z. Zeravcic, V. N. Manoharan, and M. P. Brenner, Colloquium: Toward Living Matter with Colloidal Particles, Rev. Mod. Phys. 89, 031001 (2017). 